\title{
Research
}

Jonathan Banks, Deborah J Sharp, Linda P Hunt and Julian PH Shield

\section{Evaluating the transferability of a hospital-based childhood obesity clinic to primary care:}

\section{a randomised controlled trial}

\begin{abstract}
Background

The Care Of Childhood Obesity (COCO) clinic at the Bristol Royal Hospital for Children (BRHC) uses a multidisciplinary approach comprising a consultant, dietitian, and exercise specialist. The clinic has demonstrated efficacy in managing children's weight but similar clinics are scarce in the UK
\end{abstract}

\section{Aim}

This pilot randomised controlled trial (RCT) aimed to examine the feasibility of undertaking a fully powered RCT and to gauge whether the COCO model could be effective as a nurse-led clinic in primary care settings.

\section{Design and setting}

Patients were randomised to a hospital-based childhood obesity clinic or a nurse-led clinic in a primary care setting in south-west England.

\section{Method}

Children aged $5-16$ years with a body mass index $(B M I) \geq 98$ th centile were referred by GPs to the consultant in charge of the $\mathrm{COCO}$ clinic at $\mathrm{BRHC}$. Referred children were clinically screened for suitability and invited into the study. Consenting families were randomised to $\mathrm{BRHC}$ or a primary care clinic (PCC) and offered five appointments over 12 months. Clinical effectiveness was measured by change in body mass index standard deviation score (BMI SDS) at

12 months. Other measures included: treatment adherence, quality of life (QOL), and satisfaction. Feasibility was examined by assessing referral, screening, and recruitment data.

\section{Results}

A total 152 patients were referred by GPs: 31 $(20 \%)$ were screened out; $45(30 \%)$ declined to participate. Seventy-six (50\%) patients were randomised and 68 provided baseline data (PCC = 42; $\mathrm{BRHC}=26$ ); 52 provided outcome data ( $\mathrm{PCC}=$ 29: $\mathrm{BRHC}=23$ ). Mean change in BMI SDS was PCC $-0.17(95 \%$ confidence interval $[\mathrm{Cl}]=-0.27$ to $-0.07)$; $\mathrm{BRHC}-0.15$ (95\% $\mathrm{Cl}=-0.26$ to -0.05$)$. $\mathrm{QOL}$, adherence, and satisfaction data indicated similar positive patterns in both trial arms.

\section{Conclusion}

Screening and recruitment data indicate that primary care is a clinically appropriate setting and acceptable to families. The primary clinical outcome measure (reduction in BMI SDS), along with secondary outcome measures, indicate that primary care has the potential to be effective in providing weight management for children, using the COCO model.

\section{Keywords}

obesity, childhood; general practice; primary care; body weight, management.
INTRODUCTION

While the prevalence of childhood obesity may be levelling off, ${ }^{1}$ one in five 11 year olds remain obese. A recent Cochrane Review highlighted the lack of evidence for effective weight-management programmes for childhood obesity. ${ }^{2}$

The Care Of Childhood Obesity (COCO) clinic at the Bristol Royal Hospital for Children (BRHC) is an established service that uses a multicomponent-team approach in consultations with children and families. This clinic has previously reported that $83 \%$ of children improve their body mass index standard deviation scores (BMI SDS) during 12 months' therapy. ${ }^{3}$ Similar clinic models for managing childhood obesity remain scarce, and most children with obesity have limited or no access to services able to address their health needs.

Primary care clinics (PCCs) are likely to be more accessible and would reduce pressure on greatly oversubscribed hospital clinics, allowing secondary care physicians to deal with children with associated comorbidities or requiring pharmacotherapy. However, research among primary care practitioners has identified a degree of ambivalence as to whether primary care has the resources or expertise to deal with this problem. 4,5

This pilot randomised controlled trial (RCT) examined the clinical efficacy of a nurse-led PCC compared with a consultant-led secondary care service at BRHC. The feasibility for a fully powered

J Banks, $\mathrm{PhD}$, research programme manager DJ Sharp, MD, MRCP, FRCPCH, professor of primary health care, Academic Unit of Primary Health Care, School of Social and Community Medicine; LP Hunt, PhD, senior lecturer in medical statistics, School of Clinical Sciences, University of Bristol, Bristol. JPH Shield, MD, professor of diabetes and metabolic endocrinology, School of Clinical Sciences, University of Bristol and Bristol Royal Hospital for Children, Bristol.

Address for correspondence

Deborah J Sharp, Academic Unit of Primary Health trial was assessed using a number of outcomes, including: (a) the clinical suitability of patients referred to receive PCC-based care; (b) the willingness of families to be randomised to PCCs; and (c) the degree to which families randomised to PCCs engaged with the service.

\section{METHOD}

\section{Participants and setting}

Children aged $5-16$ years with body mass index (BMI) $\geq 98$ th centile were referred to the COCO service. GPs completed an electronic referral form (The Bristol Online Obesity Screening Tooll.6 The form facilitated measurement of $\mathrm{BMI}$ and centile, and flagged up clinical conditions requiring the attention of secondary care, such as associated endocrine disorders and obesity comorbidities. Forms were reviewed by one researcher prior to study invitations being sent to eligible children. Willing participants gave verbal consent over the phone and were randomised to PCC or BRHC. Written consent and baseline data were collected at the first appointment.

Two PCCs were provided in north and south Bristol, and families could be referred from any part of Bristol, and North Somerset and South Gloucestershire Primary Care Trusts (PCTs). Families were classified as residing in either the north or south of this area, and randomised to PCC or BRHC. In north Bristol, the clinic was based in a large GP surgery, recruited via

Care, School of Social and Community Medicine University of Bristol, Canynge Hall, 39 Whately Road Bristol BS8 2SP.

E-mail: Debbie.Sharpabristol.ac.uk

Submitted: 12 April 2011; Editor's response: 31 May 2011; final acceptance: 19 July 2011. (c)British Journal of General Practice

This is the full-length article (published online 27 Dec 2011) of an abridged version published in print. Cite this article as: Br J Gen Pract 2012; DOI: 10.3399/bjgp12X616319. 


\section{How this fits in}

Given the current prevalence of childhood obesity, primary care is viewed by the NHS as an extremely important area in which to deliver obesity-management interventions. However, there remains significant ambivalence from health professionals regarding this proposition. This study demonstrates that at least $80 \%$ of children with obesity could be managed successfully in primary care using a practice-nurse-led service. Improving participant engagement in weight management remains an issue in both primary and secondary care.

the local primary care research network. The surgery was paid NHS service support cost rates for room use. The south Bristol clinic was based in a community health park, where recruitment was facilitated through the close relationship between the health park and an adjoining GP surgery which is part of the local primary care research network. As child obesity was one of the strategic targets of the health park, the room space was provided free of charge.

\section{Randomisation}

A 'minimisation method' was used to balance groups with respect to sex and age (primary or secondary school age at entry), with separate lists for the designated north and south Bristol participants. The initial allocation ratio was 1:1 but was changed to 2:1 after 5 months to ensure more patients were assigned to PCC rather than hospital, thus ensuring maximum information was obtained regarding transferring the service to PCC. New randomisation lists were set up at this point. Randomisation was undertaken by an independent statistician.

\section{Intervention}

The PCCs were led by practice nurses employed by Bristol PCT, who also provided governance for the PCCs. Nurse salaries and training costs were paid by NHS service support costs.

Nurse training involved: (1) shadowing the clinical team at the COCO outpatient clinic at BRHC on three occasions leach clinic ran for 4 hours), thus enabling the nurses to sit in with all members of the multidisciplinary team (doctor, specialist obesity nurse, dietitian, exercise specialist) on each of their three visits; (2) attendance at a one-off, secondary care workshop run by a specialist obesity nurse; (3) study packs given to nurses, to read in their own time, which included guidance on obesity management from the National Institute for Health and Clinical Excellence, ${ }^{7}$ and the Department of Health care pathway for primary care management, ${ }^{8}$ along with other literature and the background to the COCO clinic itself; 9,10 and (4) familiarisation with standard operating procedures for clinical practice written by $\mathrm{COCO}$ clinicians and research staff.

During the first year of the study, the nurse based at the north clinic moved away from the area and the nurse from the south clinic then covered both clinics for the remainder of the study. The nurse-led clinics included an exercise specialist and a dietitian, both of whom also worked in the COCO clinic at BRHC; their time at the PCCs was paid as a research cost. PCCs were largely autonomous but clinical responsibility resided with the COCO consultant and project principal investigator. In practice, clinical advice was minimal $k<10$ contacts between nurses and consultant throughout the study).

PCC patients were offered five appointments over 12 months: an initial visit and four further appointments at 3-monthly intervals. At each appointment, the family saw the practice nurse, who weighed and measured the child, plotting the data on growth charts. The nurse discussed overall progress, focusing on factors that facilitated or inhibited weight reduction. The family then saw the dietitian and exercise consultant for specialist advice.

Patients attending the BRHC clinic had an initial consultation with the $\mathrm{COCO}$ consultant. They were offered a further four COCO appointments over a 1-year period at 3monthly intervals, where they would also see a dietitian and/or exercise specialist as directed by the consultant.

Weight in kilogrammes and height in centimetres were recorded to one decimal place. In PCCs, measures were taken with portable Tanita floor scales (WB 100 S MA, Tanita Europe BV, The Netherlands) and the Seca Leicester stadiometer (Seca UK). In $\mathrm{BRHC}$, measures were recorded on Seca scales (model M861, Seca UK).

\section{Exercise and dietetic consultations}

These followed the same pattern in both arms. Exercise consultations were underpinned by a sociocognitive approach, ${ }^{11}$ taking into account social factors and specific family issues, along with the needs/wishes of the child. The emphasis was on developing strategies and activities that were enjoyable, fostered an effort-benefit return, and developed confidence that could be supported by the 


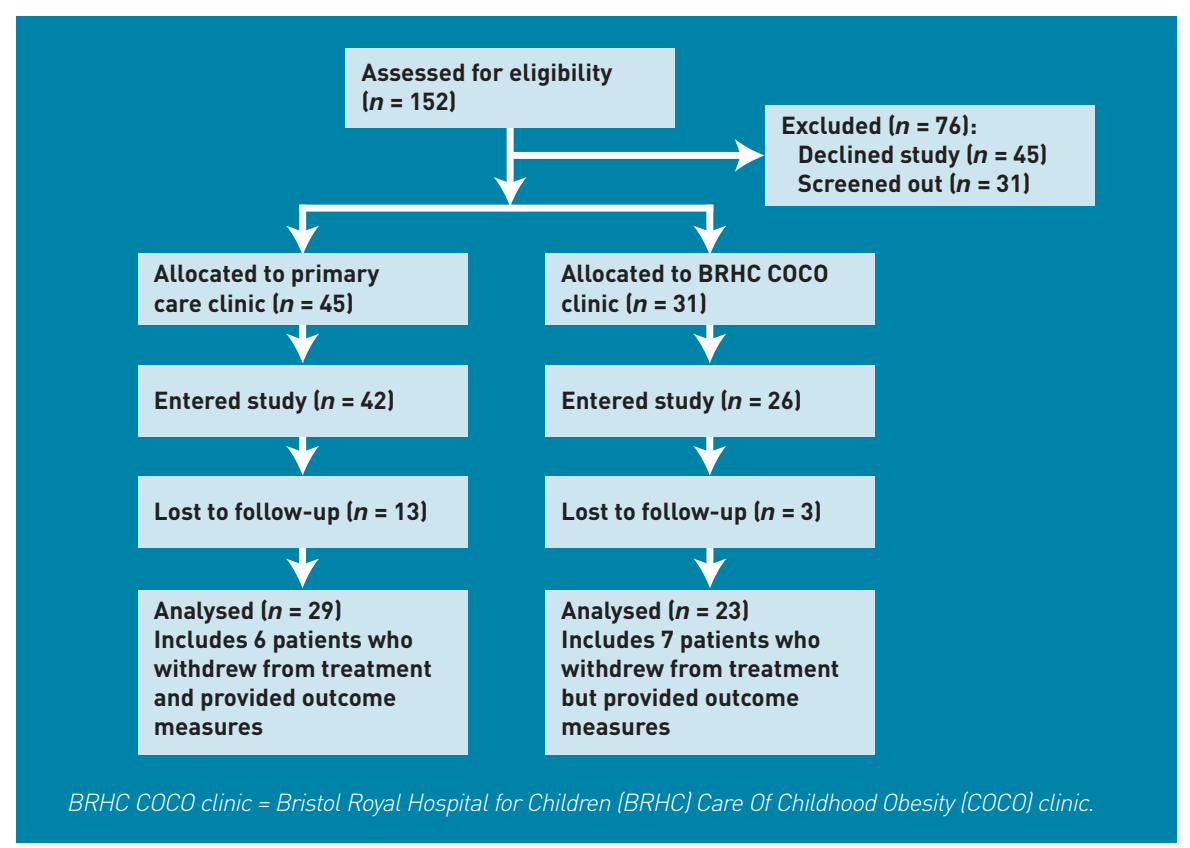

Figure 1. Consolidated Standards of Reporting Trials (CONSORT) diagram.

family. ${ }^{12}$ Age-specific approaches were used in recognition of developmental stage, with parents being the key determinants of behaviour in children aged under 10 years; the role of peers coming to the fore in early adolescence; and independent decision making in later adolescence. Dietetic consultations used a similar approach and made use of educational tools such as the 'Eatwell plate'."

\section{Outcomes}

A range of process measures were monitored, including: criteria for patients being screened out; reasons for study nonparticipation (to establish whether a significant number of families preferred to be seen in secondary care); reasons for non-adherence to treatment; and did-notattend rates between the study arms.

The primary outcome measure was change in BMI SDS at 12 months. BMI was adjusted for age and sex to give a BMI SDS

\section{Table 1. Reasons for exclusions and declining participation}

\begin{tabular}{lclc}
$\begin{array}{l}\text { Screening for clinical } \\
\text { comorbidity and } \\
\text { recruitment criteria }\end{array}$ & Excluded, $\boldsymbol{n}(\%)^{\mathbf{a}}$ & Declined participation & Declined, $\boldsymbol{n}(\%)^{\mathbf{a}}$ \\
\hline Genetic & $8(5)$ & Unable to contact & $17(11)$ \\
\hline Endocrine & $9(6)$ & Declined via contact reply form & $13(9)$ \\
\hline Type 2 diabetes, parental & $7(5)$ & Preference to see doctor/attend hospital & $6(4)$ \\
\hline Obesity comorbidity & $2(1)$ & Access problems at PCC & $4(3)$ \\
\hline Overt eating disorder & $2(1)$ & Unwilling to participate in research & $2(1)$ \\
\hline latrogenic & $1(<1)$ & Unwilling to attend any clinic & $3(2)$ \\
\hline Outside age range & $2(1)$ & & $45(30)$ \\
\hline Total & $31(20)$ & Total &
\end{tabular}

a Denominator for percentages is total referred, $n=152$. $P C C=$ primary care clinic score using the LMSGrowth, a Microsoft Excel $^{\circledR}$ add-in based on 1990 growth reference data from the Child Growth Foundation. ${ }^{14}$

Secondary outcomes were: (1) quality-oflife scores using the Pediatric Quality of Life Scale (PedsQL); ${ }^{15}$ (2) satisfaction with care using an adapted instrument, developed from a similar study in primary care, ${ }^{16}$ and the General Practice Assessment Questionnaire. ${ }^{17}$ Satisfaction was measured in relation to 'consultations' (six-item scale that considered interaction of clinical team with participants); 'appointments' (fouritem scale that considered organisation of appointments including waiting time); and 'access' (six-item scale that considered how easy the clinics were to access in terms of travel and location). Each scale was scored from $1=$ excellent to $6=$ very poor.

\section{Sample size}

As this was a feasibility study, it was not powered to achieve statistical significance for the primary clinical outcome. It was anticipated that 100 participants could be recruited over a 1-year period.

\section{Statistical methods}

The primary clinical outcome was the change in BMI SDS at 12 months. Mean (standard deviation) of the changes was calculated for each group, together with the difference between the mean changes and a two-sided $95 \%$ confidence interval (CI) for the difference. Linear models were used to explore the influence of other factors on the group mean difference. 
Table 2. Allocation and baseline comparisons

\begin{tabular}{|c|c|c|}
\hline Allocation $(n=76)$ & $\operatorname{BRHC}(n=31)$ & $\operatorname{PCC}(n=45)$ \\
\hline North:south & $16: 15$ & $21: 24$ \\
\hline Male:female & $13: 18$ & $16: 29$ \\
\hline Primary:secondary school & $15: 16$ & $22: 23$ \\
\hline Baseline $(n=68)^{a}$ & $\operatorname{BRHC}(n=26)$ & $\operatorname{PCC}(n=42)$ \\
\hline Age in years: mean (SD), range & $11.5(2.5), 5.8$ to 14.9 & $11.4(2.8), 5.7$ to 17.0 \\
\hline BMI SDS: mean (SD), range & $2.86(0.40), 2.15$ to 3.60 & 3.17 (0.57), 2.05 to 4.74 \\
\hline
\end{tabular}

With baseline differences in BMISDS observed between the groups (Table 2), 12month comparisons were explored further using covariate adjustment for baseline, rather than calculating simple changes; results were very similar (data are not shown). A $\chi^{2}$ test was used to compare withdrawal rates between the two groups, and a two-sample $t$-test was used to compare change in mean PedsQL.

\section{RESULTS}

Recruitment took place between April 2008 and May 2009. Figure 1 shows participant flow through the trial.

\section{Recruitment}

One-hundred and fifty-two children were referred by GPs. Thirty-one (20\%) patients were screened out: 29 for suspected clinical comorbidities and two were outside the age range for the study (Table 1, Figure 1). Between 1 April 2008 and 1 November 2008, patients whose referral form indicated parental type 2 diabetes were not invited into the study but received usual care, which included a full oral glucose tolerance test at BRHC. Following a clinical review of the COCO clinic, the investigators concluded that the risk of type 2 diabetes for such children was minimal, and children referred subsequently were invited into the study and, if allocated to PCC, given an appointment at $\mathrm{BRHC}$ for an oral glucose tolerance test. Two patients allocated to PCC subsequently had negative tests and continued in the PCC arm.

One hundred and twenty-one (80\%) patients were suitable for primary care management and invited into the study. Forty-five families (30\%) declined to participate (Table 1). The majority were categorised as: unable to contact $(n=17$, $11 \%)$, or declined via the contact reply form without giving a reason $(n=13 / 9 \%)$. Six $(4 \%)$ stated a clear preference to attend hospital.

Allocation and baseline comparisons The distribution between study arms (PCC =

Table 3. Changes in body mass index standard deviation scores (BMI SDS)

\begin{tabular}{lccccc} 
& \multicolumn{7}{c}{ BMISDS, $\boldsymbol{n} \mathbf{( \% )}$} \\
\cline { 2 - 6 } & $\begin{array}{c}\text { Reduced more } \\
\text { than } \mathbf{0 . 5}\end{array}$ & $\begin{array}{c}\text { Reduced between } \\
\mathbf{0 . 2 5} \text { and } \mathbf{0 . 5}\end{array}$ & $\begin{array}{c}\text { Reduced between } \\
\mathbf{0} \text { and } \mathbf{0 . 2 5}\end{array}$ & $\begin{array}{c}\text { Increased between } \\
\mathbf{0}^{\mathbf{a}} \text { and } \mathbf{0 . 2 5}\end{array}$ & $\begin{array}{c}\text { Increased more } \\
\text { than } \mathbf{0 . 2 5 ^ { \mathbf { a } }}\end{array}$ \\
\hline Total $(n=52)$ & $6(12)$ & $9(17)$ & $25(48)$ & $11(21)$ & $1(2)$ \\
\hline $\mathrm{BRHC}(n=23)$ & $2(9)$ & $5(22)$ & $11(48)$ & $5(22)$ & $0(0)$ \\
\hline $\mathrm{PCC}(n=29)$ & $4(14)$ & $4(14)$ & $14(48)$ & $6(21)$ & $1(3)$ \\
\hline
\end{tabular}

${ }^{a}$ Range includes these end points. BRHC = Bristol Royal Hospital for Children. $P C C=$ primary care clinic.

\section{Table 4. Comparison of BMI SDS changes between BRCH and PCC (North and South Bristol)}

\begin{tabular}{|c|c|c|c|c|c|c|}
\hline \multirow[b]{2}{*}{ Mean change } & \multicolumn{2}{|c|}{$\begin{array}{c}\text { North and } \\
\text { South combined }\end{array}$} & \multicolumn{2}{|c|}{ South } & \multicolumn{2}{|c|}{ North } \\
\hline & $\begin{array}{l}\text { BRHC } \\
(n=23)\end{array}$ & $\begin{array}{c}\text { PCC } \\
(n=29)\end{array}$ & $\begin{array}{l}\text { BRHC } \\
(n=11)\end{array}$ & $\begin{array}{c}\text { PCC } \\
(n=15)\end{array}$ & $\begin{array}{l}\text { BRHC } \\
\text { (n=12) }\end{array}$ & $\begin{array}{c}\text { PCC } \\
(n=4)\end{array}$ \\
\hline BMI SDS (SD) & $-0.15(0.25)$ & $-0.17(0.26)$ & $-0.09(0.16)$ & $-0.26(0.26)$ & $-0.21(0.30)$ & $-0.08(0.24)$ \\
\hline two-sided $95 \% \mathrm{Cl}$ & -0.26 to -0.05 & -0.27 to 0.07 & & & & \\
\hline \multirow{2}{*}{\multicolumn{2}{|c|}{$\begin{array}{l}\text { Mean difference (SE) BRHC minus PC } \\
\text { two-sided } 95 \% \mathrm{Cl}\end{array}$}} & $\begin{array}{ll}\mathrm{CC} & 0.02\end{array}$ & $(0.07)$ & & & \\
\hline & & -0.12 & to 0.17 & & & \\
\hline
\end{tabular}


Table 5. Mean satisfaction scores for BRHC and PCCa

\begin{tabular}{llc} 
& BRHC mean (SD) & PCC mean (SD) \\
\hline Consultations & $2.03(0.94), n=16$ & $1.59(0.77), n=22$ \\
\hline Appointments & $2.85(0.89), n=17$ & $1.65(0.62), n=22$ \\
\hline Access/convenience & $2.84(0.83), n=17$ & $1.91(0.76), n=22$ \\
\hline a $n$ sow scores indicate high satisfaction: $1=$ excellent to $6=$ very poor. BRHC $=$ Bristol Royal Hospital for Children. \\
PCC = primary care clinic.
\end{tabular}

\section{Funding}

This paper presents independent research commissioned by the National Institute for Health Research (NIHR) under its Research for Patient Benefit Programme Reference Number PB-PG-0706-10090. The views expressed are those of the author(s) and not necessarily those of the NHS, the NIHR or the Department of Health.

\section{Ethical approval}

Ethical approval for the study was granted by Southmead Research Ethics Committee on 18/07/2007; MREC No: 07/Q2002/35.

\section{Trial details}

The study was registered at ClinicalTrials.gov NCT00536536. The design, conduct, and reporting of the trial follows Consolidated Standards of Reporting Trials (CONSORT) guidelines. ${ }^{24}$

\section{Provenance}

Freely submitted; externally peer reviewed.

\section{Competing interests}

The authors have declared no competing interests.

\section{Acknowledgements \\ The authors would like to acknowledge the help and support of all the clinical staff involved in the study: Sarah Bergin, Nikki Davis, Shelley Easter, Anna Ford, Mr Riyaz Jamal, Laura James, Christina Lampey, Christine Lee, Pauline Taggart, and Jessica Williams. We are thankful to the Bristol Royal Hospital for Children and the two primary care sites for hosting the study, as well as the patients and families who participated in the research.}

\section{Discuss this article}

Contribute and read comments about this article on the Discussion Forum: http://www.rcgp.org.uk/bjgp-discuss
45 and $\mathrm{BRHC}=31$, Table 2) reflects the change in allocation ratio from 1:1 to $2: 1$ introduced after 5 months. Eight of the 76 children randomised (three PCC and five $\mathrm{BRHC}$ ) did not attend their first appointment and declined to enter the trial formally. Of the families randomised to PCC, 15\% (3/21) were registered with a GP at the north clinic practice, and 33\% (8/24) were registered with one of the two GP practices co-located within the community health park where the south clinic was based.

Table 2 shows baseline comparison of the two arms. Of those providing baseline data, children referred to PCCs had higher mean BMI SDS than those referred to BRHC.

\section{Outcome data}

The primary outcome was change in BMI SDS between baseline and the fifth appointment (12 months from baseline). Outcome data were collected from 52 patients (23/26 BRHC and 29/42 PCC, 76\% of those providing baseline data). Data include 13 patients who withdrew from treatment but agreed to have a final outcome measure taken at home, 1 year after baseline measures. Analysis was by intention to treat.

The majority of children in both arms, 40/52 (77\%) improved their BMI SDS scores, with $15(29 \%)$ showing reductions of more than 0.25 BMI SDS (Table 3).

At $\mathrm{BRHC}$, the mean $\mathrm{BMI}$ SDS reduction was 0.15, and in PCC 0.17 (Table 4); the difference in means was 0.02 (two-sided $95 \% \mathrm{Cl}=-0.12$ to 0.17$)$, providing no evidence that PCC was inferior to BRHC. The group difference did not change substantially when adjusted for randomisation period (before/after the change-over from 1:1 to 2:1), or for sex/age group. However, some variation was observed between the two PCCs, with the south Bristol clinic showing the best mean improvement in BMI SDS of -0.26 BMI SDS.

\section{Treatment adherence and attendance}

Both arms experienced high levels of nonadherence, with nearly half of those starting treatment withdrawing $(29 / 68,43 \%)$.
Withdrawals were higher in PCC (19/42 = $45 \%)$ compared with BRHC (10/26 = 38\%) but the difference was not statistically significant $(P=0.77)$. Phone interviews with non-adhering families highlighted a number of issues. Motivation was the most prominent theme: parents struggled to motivate children between appointments, often leading to conflict between parent and child, thus disrupting family life. Some families felt clinic advice to be impractical or overambitious, and some felt it was not age appropriate. Families also cited family events that overrode their commitment to participate in the programme.

The overall did-not-attend rate (total didnot-attend/total appointments offered) was $23 \%$, which was similar in both arms (BRHC $=24 \% ; \mathrm{PCC}=22 \%$ ).

\section{Quality of life}

PedsQL scores rose in both arms over the 12 months: 10 points in PCC $(95 \% \mathrm{Cl}=3$ to 18 points, $n=23$ ) compared with 8 points in BRHC $(95 \% \mathrm{Cl}=-2$ to 18 points, $n=14)$, two-sample $t$-test $P=0.65)$.

\section{Satisfaction}

The satisfaction questionnaire considered the consultation, timing, and frequency of appointments, and access and convenience of the clinic. The PCCs scored slightly higher for each aspect of satisfaction: consultations, appointments, and access (Table 5), although all mean scores were between 1 and 3 , equivalent to ratings from 'excellent' to 'good'.

\section{DISCUSSION}

\section{Summary}

The original target was to recruit 100 children into the study. With 76 patients randomised and 68 starting the trial, this original target was not met, but the 152 children referred provided a substantive body of data by which to assess the referral and recruitment process. GP assessment for referral using the Bristol Online Obesity Screening Tool showed that the majority of patients referred $(80 \%)$ were suitable for primary care weight management and so they were invited into the study. Forty-five (30\%) patients referred declined to participate. However, it should be noted that only 21 (14\%) expressed a clear preference for the usual care pathway at BRHC Isix preferred to see a doctor, 13 declined without reason, and two were unwilling to participate in research). This does not suggest widespread opposition to a primary-care-based service.

The 1-year reduction in children's BMI 
SDS does not appear compromised, with similar results in PCC compared to BRHC. The overall BMI SDS improvements are better than those described in the recent Cochrane meta-analysis of randomised trials in childhood obesity. ${ }^{2}$ However, a BMI SDS change of between -0.15 and -0.17 is too small to be certain of an improvement in metabolic health, which requires a change of $\geq-0.25$. 18

Obesity in children is associated with low self-esteem, behavioural problems, ${ }^{19}$ and bullying. ${ }^{20}$ The data from this study suggest an equivalent improvement in quality of life between PCC and BRHC over the course of the trial.

The PCCs were unable to improve on the non-adherence rate in BRHC, and qualitative data (paper in preparation) indicated non-adherence was not linked to clinic type. The main issue was that patients lacked motivation and proved unresponsive to the efforts of clinic staff and parents. Alongside this, it is worth noting that a number of families stopped attending due to individual/family events, indicating that obesity management is perceived as an optional service, with non-attendance being thought to have little medical consequence. The reasons provided for non-adherence in the present study, particularly patient unpreparedness for change, were noted in another report by Barlow and Ohlemeyer examining non-adherence to a weightmanagement intervention programme. ${ }^{21}$ However, those families completing treatment in the current study were satisfied with service provision in either care site.

\section{Strengths and limitations}

At a time when policy makers and practitioners are searching for effective methods to reduce the health burden represented by childhood obesity, this study has taken a proven model of care and adapted it to a primary care setting, with promising results.

The authors recognise that as a pilot RCT the study is not statistically powered and the data must therefore be interpreted with caution. The authors are also aware that establishing two primary care clinics to cover the whole of Bristol and neighbouring PCTs does not test the locality aspect of the clinics, as only a small proportion of patients were registered with the practices where the clinics were sited. It is entirely plausible that clinics that are recognised by patients as local could improve adherence, and this is something that should be factored into a full trial design.

\section{Comparison with existing literature}

An Australian PCC-based trial, where treatment was based on four GP consultations with each family and child over a 12-week period, was unable to show a significant reduction in BMI compared with controls, and overall BMI change (not BMI SDS) was -0.12 at 12 months. ${ }^{22}$ Qualitative research has found a degree of uncertainty among primary care practitioners about taking on child obesity treatment. 4,5 However, the present results do suggest a potential role for primary care, utilising nursing staff instead of doctors in locality-based, community clinics serving groups of practices, with overall clinical responsibility residing with the original referring GP.

A recent study has evaluated the MEND (Mind, Exercise, Nutrition ... Do it!) programme, ${ }^{23}$ which runs from local authority community settings as well as primary care sites. The programme is based on 18 sessions of group advice and exercise over 9 weeks. BMI SDS reductions in the intervention group were significantly better than in the control group, with an overall reduction of 0.24 . This study highlights the potential for managing obesity in community settings. However, this programme involves a much higher level of contact with the patient and family over a shorter period, and its success is dependent on group participation, which will not be suitable for all children.

\section{Implications for practice and research}

The data from this study suggest that there is scope to develop child obesity services in primary care. The prevalence of child obesity, along with the growing recognition that it is a critical public health problem, means that the few existing secondary care services are likely to be overwhelmed by demand. The study shows that, with appropriate training and support, primary care has the potential to be an effective site for weight management for children. Improving the proportion of suitable patients engaging and accessing services, patient retention, and the identification of interventions accruing a proportionally larger effect on BMI SDS remain unresolved issues for both primary and secondary care. 


\section{REFERENCES}

1. The Information Centre for Health and Social Care. National child measurement programme: England 2008/09 school year. Leeds: The Information Centre for Health and Social Care, 2009.

http://www.ic.nhs.uk/webfiles/publications/ncmp/ncmp0809/NCMP_England_ 2008_09_school_year_report_2.pdf laccessed 4 Aug 2011).

2. Oude Luttikhuis $H$, Baur $L$, Jansen $H$, et al. Interventions for treating obesity in children. Cochrane Database Syst Rev 2009; (1): CD001872.

3. Sabin MA, Shield JPH. Childhood obesity. Front Horm Res 2008; 36: 85-96.

4. Walker $\mathrm{O}$, Strong M, Atchinson $\mathrm{R}$, et al. A qualitative study of primary care clinicians' views of treating childhood obesity. BMC Fam Pract 2007; 8: 50-57.

5. Turner KM, Shield JPH, Salisbury C. Practitioners' views on managing childhood obesity in primary care: a qualitative study. Br J Gen Pract 2009; 59(568): 856-886.

6. Owen SE, Sharp DJ, Shield JPH. The Bristol Online Obesity Screening Tool: experience of using a screening tool for assessing obese children in primary care. Prim Health Care Res Dev 2011; 12(4): 293-300.

7. National Institute for Health and Clinical Excellence. Obesity: the prevention, identification, assessment and management of overweight and obesity in adults and children. CG43. London: National Institute for Health and Clinical Excellence, 2006. http://www.nice.org.uk/guidance/CG43 laccessed 4 Aug 2011).

8. Department of Health. Obesity care pathway and your weight, your health. London: Department of Health, 2006.

http://www.dh.gov.uk/PublicationsAndStatistics/Publications/PublicationsPolic yAndGuidance/PublicationsPolicyAndGuidanceArticle/fs/en?CONTENT_ID=41 34408\&chk=Sq/wNd laccessed 4 Aug 2011).

9. Sabin MA, Ford A, Hunt L, et al. Which factors are associated with a successful outcome in a weight management programme for obese children? J Eval Clin Pract 2007; 13(3): 364-368.

10. Shield J, Summerbell C. Obesity in childhood. In: Williams G, Fruhbeck G (eds). Obesity: science to practice. Chichester: Wiley-Blackwell, 2009; 509-533.

11. Bandura A. Social foundations of thought and action: a social cognitive theory. Englewood Cliffs, NJ: Prentice Hall, 1986.
12. Biddle SJ, Mutrie N. Psychology of physical activity: determinants, well-being and interventions. 2nd edn. London: Routledge, 2008.

13. NHS Choices. The eatwell plate.

http://www.eatwell.gov.uk/healthydiet/eatwellplate/ laccessed 4 Aug 2011).

14. Cole TJ, Freeman JV, Preece MA. Body mass index reference curves for the UK, 1990. Arch Dis Child 1995; 73(1): 25-29.

15. Varni JW, Seid M, Kurtin PS. PedsQL 4.0: reliability and validity of the pediatric quality of life inventory version 4.0 generic core scales in healthy and patient populations. Med Care 2001; 39(8): 800-812.

16. Salisbury C, Noble A, Horrocks $S$, et al. Evaluation of a general practitioner with a special interest service for dermatology: randomised controlled trial. BMJ 2005; 331(7530): 1441-1444

17. University of Cambridge. The General Practice Assessment Questionnaire (GPAQ). http://www.gpaq.info/ laccessed 4 Aug 2011).

18. Ford AL, Hunt LP, Cooper A, Shield JP. What reduction in BMI SDS is required in obese adolescents to improve body composition and cardiometabolic health? Arch Dis Child 2010; 95(4): 256-261.

19. Reilly JJ, Methven E, McDowell ZC, et al. Health consequences of obesity. Arch Dis Child 2003; 88(9): 748-752.

20. Janssen I, Craig WM, Boyce WF, Pickett W. Associations between overweight and obesity with bullying behaviours in school-aged children. Pediatrics 2004; 113(5): 1187-1194.

21. Barlow $\mathrm{S}$, Ohlemeyer $\mathrm{C}$. Parent reasons for nonreturn to a pediatric weight management programme. Clin Pediatr 2006; 45(4): 355-360.

22. Wake $M$, Baur $L A$, Gerner $B$, et al. Outcomes and costs of primary care surveillance and intervention for overweight or obese children: the LEAP 2 randomised controlled trial. BMJ 2009; 339: b3308.

23. Sacher PM, Kolotourou M, Chadwick PM, et al. Randomized controlled trial of the MEND program: a family-based community intervention for childhood obesity. Obesity 2010; 18(S2): S1-S7.

24. Altman DG, Schulz KF, Moher D, et al; CONSORT GROUP (Consolidated Standards of Reporting Trials). The revised CONSORT statement for reporting randomized trials: explanation and elaboration. Ann Intern Med 2001; 134(8): 663-694. 